\title{
Escherichia coli and Klebsiella pneumonia as the Most Common Bacteria Causing Catheter Associated Urinary Tract Infection
}

\author{
Dwiyanti Puspitasari ${ }^{1}$, Edward Adijaya Rusli ${ }^{2}$, Dominicus Husada ${ }^{1}$, Leny Kartina ${ }^{1}$ \\ ${ }^{1}$ Department of Pediatric, Dr Soetomo General Hospital/Faculty of Medicine, Universitas Airlangga, Surabaya, Indonesia \\ ${ }^{2}$ Faculty of Medicine, Universitas Airlangga, Surabaya, Indonesia
}

Background: Healthcare-Associated Infections (HAls) are the result of a reaction between taint agents that infected the patient when the patient is hospitalized. A Study from The Center for Disease Control and Prevention shows that most HAls in hospital are urinary tract infection, most of the infection incident in patient are caused by catheter. Catheter indwelling is notable in medical sphere. This study aimed to inquire case number of Catheter-Associated Urinary Tract Infection (CAUTI) in Dr. Soetomo General Hospital, the feature of CAUTI patients, the type of bacteria that cause CAUTI, and what is the relation among sex and bacteria colony.

Materials and Methods: An analytic observational study with the population of pediatric hospitalized patients of Dr. Soetomo General Hospital was conducted in January-December 2017. Samples collected were positive urine culture from pediatric hospitalized patients. Information regarding the bacteria that cause CAUTI, gender, and length of catheter usage were collected.

Results: There were total 140 samples of positive urine culture in pediatric patient, and $38.5 \%$ was diagnosed as CAUTI. Overall CAUTI was often found in male subjects (51.9\%), and similar with $\leq 1$-year old patients which also often found in male subjects $(60.8 \%)$. The highest length of catheter usage was 3-5 days (42.5\%). All subjects had fever as a clinical sign and $83.3 \%$ had suprapubic pain. Escherichia coli and Klebsiella pneumoniae infections were highly discovered. There was an association between gender and urine culture colony count ( $p=0.02)$.

Conclusion: CAUTI is commonnly found in Dr. Soetomo General Hospital, and two bacteria that cause the most infection were E. coli and K. pneumoniae.

Keywords: catheter, urinary tract infection, healthcare associated infection

\section{Introduction}

Healthcare-Associated Infections or HAIs often become a common factor causing morbidity and mortality in the world. Based on World Health Organization (WHO), HAIs can be interpreted as an infection that patients get while receiving hospital treatment and the infection is not found when the patient came. A Study conducted by WHO showed that in 1995-2010, The prevalence of HAIs in Indonesia was as many as $7.1 \%{ }^{1}$

Date of submission: August 5, 2021

Last Revised: September 17, 2021

Accepted for publication: September 19, 2021

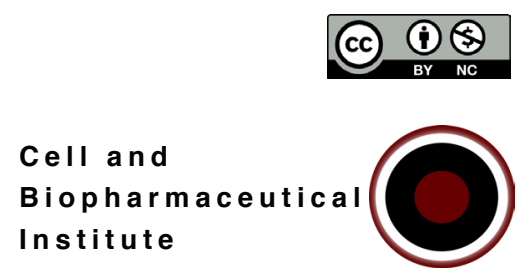

Corresponding Author:

Edward Adijaya Rusli

Faculty of Medicine, Universitas Airlangga

Jl. Mayjen Prof. Dr. Moestopo No.47, Surabaya, Indonesia

e-mail: rusliedo@ymail.com

Cell and

Institute 
According to The Centers for Disease Control and Prevention (CDC) in America in 2007, HealthcareAssociation Infection in urinary tract infection reached $40 \%{ }^{2}$ From $40 \%$, 95\% of urinary tract infection occurs in inpatient room because of the catheter installation. The indwelling catheter is an important component in medical. $^{2}$

Catheter associated urinary tract infection (CAUTI) is the largest nosocomial infection around the world that reach up to $30-40 \%$. According to CDC, each patient using catheter for 48 hours and infected by urinary tract infection is CAUTI. According to The National Health Care Safety Network (NSHN), every tube that entered the bladder through The urethra is included in indwelling catheters except the suprapubic catheter and nephrostomy tube. ${ }^{3}$

Catheter usage is increasing the risk of CAUTI incidence. This infection is occurred because of the biofilm that gathers the bacteria inside and outside the catheter surface right after the catheter entered. Catheter usage also reduced body ability to eliminate bacteria from the lower urinary tract. ${ }^{3}$

Urinary tract infection prevention efforts due to catheterization are being focused on good catheter installation techniques and the right indication. Catheter installation must be done by trained medical personnel and using recommended aseptic techniques, using sterile equipment. Urinary tract infection (UTI) due to catheterization is also often caused by improper indwelling (long-term) catheters such as inpatient with urinary incontinence without indication of catheter placement, for patient's convenience, at the patient request or for monitoring urine count noncritical patient. Catheter indwelling should be removed immediately if there is no more indication. Alternatively, intermittent catheter or suprapubic catheter can be used with UTI risk due to smaller catheterization. Other prevention efforts should be more considered such as the treatment of urethral meatus, taking exact urine specimens, when appropriate catheter replacement and education for the patient , and their families. ${ }^{4}$

Young patients who have an indwelling urethral catheter for five days or longer will easily develop bacteriuria or candiduria in almost $50 \%$ of the cases $^{4}$, however data about CAUTI in Indonesian children was limited prior to this research. Therefore, we conducted a study of CAUTI in hospitalized children at Dr. Soetomo General Hospital, a tertiary referral hospital in Surabaya, Indonesia.

\section{Materials and methods}

This was an analytic cross-sectional study to describe the characteristics of CAUTI and number of incidents based on pediatric inpatients at Dr. Soetomo General Hospital. The research data were obtained from patient medical records of pediatric inpatients in Dr. Soetomo General Hospital in the period of January $1^{\text {st }}$ to 31 December $31^{\text {st }}, 2017$. This study had been approved by the Ethical Committee of Dr. Soetomo General Hospital (No. 707/ Panke.KKE/XE/2017).

\section{Sample Collection}

The inclusion criteria were pediatric inpatient subjects at Dr. Soetomo General Hospital, had catheter placement with urine culture positive after more than $2 \times 24$ hours. Subjects which clasified for having CAUTI according to the CDC were subject whose at least 1 clinical symptom without other cause: fever $>38^{\circ} \mathrm{C}$, suprapubic tenderness, positive nitrite, or pyuria. Bacteria found in unspun urine with gram stain. Subjects had positive urine culture of bacteria with bacterium of $\geq 10^{5} \mathrm{CFU} / \mathrm{mL}$ or $>10^{3}$ to $<10^{5} \mathrm{CFU} /$ $\mathrm{mL}$. Subjects were excluded if they meet the criteria as follows: incompleted medical records (catheter placement date, patient clinical signs and symptoms, culture urine date records), patient with positive urine culture but medical record not found, urine culture were done $<2 \times 24$ hours after catheter placement, had urinary tract infection already before catheter placement.

Criteria for CAUTI-1A patient must has a urine culture with bacterium colony count of $\geq 10^{5} \mathrm{CFU} / \mathrm{mL}$ and patient must meet at least one or more clinical symptoms such as fever $>38^{\circ} \mathrm{C}$, suprapubic tenderness, costovertebral pain whereas CAUTI-2A with bacterium colony count in positive urine culture $\geq 10^{3}-<10^{5} \mathrm{CFU} / \mathrm{mL}$ and at least one or more clinical symptoms same as CAUTI-1A but with addition of one or more positive laboratory results for nitrite positive, pyuria, microorganism seen on gram unspun urine examination.

\section{Statistical Analysis}

Data was anaylzed to find the exact number of cases of CAUTI. The Chi-square test was used to find the correlation between gender and bacteria colony. Data processing was performed using Statistical Package for Social Sciences for Windows (SPSS) version 25.0 (IBM Corporation, Armonk, NY, USA) with $p<0.05$ considered significant. 


\section{Results}

As much as 140 total samples with positive urine culture via catheter were obtained from the secondary data collection from pediatric inpatient of Dr. Soetomo General Hospital in 2017. From 140 samples, 86 samples were excluded because of following reasons: 52 samples with incompleted or missing medical records, and 34 samples did not meet the inclusion criteria. Only 54 samples met the inclusion criteria. Incident rate could not be calculated because the denumerator cannot be found, therefore the CAUTI incidence rate in 2017 were 54 .

\section{Clinical Characteristics of Subjects}

The highest CAUTI case was at the age of $\leq 1$ year $(42.6 \%)$, followed by age 2-4 years at (22.2\%), age 11-16 years at $(20.4 \%)$, and the least was at the age of $5-10$ years $(14.8 \%)$ (Table 1).

From the distribution of subjects based on gender, it was found that more male subjects were infected with CAUTI (51.9\%) compared to female subjects (48.1\%). Meanwhile the sample distribution based on gender and age $\leq 1$ year found that 14 male subjects $(60.8 \%)$ and 9 female subjects (39.1\%) were infected with CAUTI.

The results of this data showed that all CAUTI subjects in this research had fever (100\%) and some subjects had suprapubic symptoms (83.3\%) which were the CDC criteria to be diagnosed as CAUTI.

\section{Catheter Usage Duration in CAUTI Patients}

Duration frequency on catheter placement in patient of CAUTI amounted to 54 subjects (Table 1). A total of 54 subjects were screened, there were 23 subjects who had catheter application for 3-5 days (42.6\%), 22 subjects had catheter application for 6-8 days (40.7\%), 4 subjects had catheter application for 9-11 days (7.4\%) and 5 subjects had catheter placed for 12 days (9.3\%). Data showed that CAUTI patients with catheter application of 3-5 days had highest numbers compared to another group.

\section{Frequency of CAUTI 1A and CAUTI 2A}

Frequency of CAUTI types was shown in Table 1, 34 subjects included in CAUTI-1A type (63\%) and 20 subjects included in CAUTI-21 type (37\%). CAUTI-1A patient has a urine culture with bacterium colony count of $\geq 10^{5} \mathrm{CFU} /$ $\mathrm{mL}$, whereas CAUTI-2A with bacterium colony count in positive urine culture $\geq 10^{3}-<10^{5} \mathrm{CFU} / \mathrm{mL}$ and at least one
Table 1. Clinical characteristics of CAUTI subjects in Dr. Soetomo General Hospital $(n=54)$.

\begin{tabular}{|c|c|c|}
\hline Characteristics & $\mathbf{n}$ & $\%$ \\
\hline \multicolumn{3}{|l|}{ Age } \\
\hline$\leq 1$-year-old & 23 & 42.6 \\
\hline 2-4-year-old & 12 & 22.2 \\
\hline 5-10-year-old & 8 & 14.8 \\
\hline 11-16-year-old & 11 & 20.4 \\
\hline \multicolumn{3}{|l|}{ Gender } \\
\hline Male & 28 & 51.9 \\
\hline Female & 26 & 48.1 \\
\hline \multicolumn{3}{|l|}{ Having Fever $>38^{\circ} \mathrm{C}$} \\
\hline Yes & 54 & 100.0 \\
\hline No & 0 & 0 \\
\hline \multicolumn{3}{|c|}{ Having Suprapubic Tenderness } \\
\hline Yes & 45 & 83.3 \\
\hline No & 9 & 16.7 \\
\hline \multicolumn{3}{|l|}{ Catheter Usage Duration } \\
\hline 3-5 days & 23 & 42.6 \\
\hline 6-8 days & 22 & 40.7 \\
\hline 9-11 days & 4 & 7.4 \\
\hline$>12$ days & 5 & 9.3 \\
\hline \multicolumn{3}{|l|}{ CAUTI Types } \\
\hline CAUTI 1A & 34 & 63.0 \\
\hline CAUTI 2A & 20 & 37.0 \\
\hline
\end{tabular}

or more clinical symptoms same as CAUTI-1A but with addition of one or more positive laboratory results for nitrite positive, pyuria, microorganism seen on gram unspun urine examination. The data above showed that CAUTI-1A was more commonly found than CAUTI-2A.

\section{Relation of Gender and Bacteria Colony Count in CAUTI Subjects}

The gender relation with bacteria colony count could be seen in (Table 2). From the results of the table above, it can be concluded that the $p<0.05$, which means that there was an association between gender and the number of colonies.

\section{Bacteria Causing CAUTI}

Among 54 study subjects were classified in CAUTI categories based on the bacteria causing CAUTI (Table 3 ). Based on positive urine culture results, 24 subjects had Escherichia coli positive urine culture (44.4\%), 9 subjects had Klebsiella pneumoniae positive (16.7\%), 7 subjects 
Table 2. Relation of gender and bacteria colony count in CAUTI subjects.

\begin{tabular}{lcccc}
\hline \multirow{2}{*}{ Gender } & \multicolumn{2}{c}{ Colony Count } & Total & $\boldsymbol{p}$-value \\
\cline { 2 - 3 } & $\geq 10^{5}$ & $\geq 10^{3}-<10^{5}$ & & \\
\hline Male & 17 & 11 & 28 & \\
Female & 23 & 3 & 26 & 0.02 \\
Total & 40 & 14 & 54 & \\
\hline
\end{tabular}

had Enterococcus faecalis positive (13.0\%). These data showed that there were 11 subjects of gram-positive bacteria $(20.3 \%)$ and there were 43 subjects of gram-negative bacteria $(79.3 \%)$.

\section{Multiple Drug-resistant Organism (MDRO) Bacteria as the Cause of CAUTI}

Out of 54 causative bacteria subjects of CAUTI (Table 4), were obtained for 26 bacterial subject are classified to MDRO, while 16 subjects Escherichia coli Extendedspectrum beta-lactamase (ESBL) positive $(61.5 \%)$. These data showed that the most causative bacteria of CAUTI that classified to MDRO are Escherichia coli ESBL and Klebsiella pneumoniae ESBL.

\section{Discussion}

The results of this study found that high number in patient were found in subjects aged $\leq 1$ year, followed by $2-4$ years, 11-16 years and the lowest number was subjects aged 5-10 years old. In subjects aged $\leq 1$ year, one subject had imperfect immunity condition, especially immature macrophage. When a child still in the first-trimester womb, mature neutrophil appeared and the number increasing till birth. After birth, neutrophil was back to normal point with a very low killing bacteria function especially in inflammation reaction, this can cause children under 1 year are prone to infection. ${ }^{5}$

Although theoretically, the younger the patient then the affected risk increases, but this research found that

Table 3. Bacteria gram (+) and (-) that cause CAUTI $(n=54)$.

\begin{tabular}{lcc}
\hline \multicolumn{1}{c}{ Type Mycobacteria Gram (-) } & $\begin{array}{c}\text { Positive Culture } \\
\text { Count }\end{array}$ & \% \\
\hline Escherichia coli & 24 & 44.4 \\
Klebsiella pneumoniae & 9 & 16.7 \\
Klebsiella oxytoca & 1 & 1.8 \\
Pseudomonas aeruginosa & 1 & 1.8 \\
Enterobacter cloacae & 3 & 5.5 \\
Enterobacter aerogenes & 1 & 1.8 \\
Elizabethkingia meningoseptica & 1 & 1.8 \\
Burkholderia cepacia & 1 & 1.8 \\
Acinetobacter baumannii & 2 & 3.7 \\
\hline & Positive Culture & $\%$ \\
Type Mycobacteria Gram (+) & Count & 13.0 \\
\hline Enterococcus faecalis & 7 & 1.8 \\
Staphylococcus epidermidis & 1 & 1.8 \\
MRSA & 1 & 3.7 \\
Corynebacterium spp. & 2 &
\end{tabular}




\begin{tabular}{|c|c|c|}
\hline Mycobacteria Type & $\mathbf{n}$ & $\%$ \\
\hline Escherichia coli $\mathrm{ESBL}$ & 16 & 61.5 \\
\hline Klebsiella pneumoniae ESBL & 5 & 19.2 \\
\hline Acinetobacter baumannii & 2 & 7.7 \\
\hline Klebsiella oxytoca ESBL & 1 & 3.8 \\
\hline Pseudomonas aeruginosa & 1 & 3.8 \\
\hline$M R S A$ & 1 & 3.8 \\
\hline
\end{tabular}

the lowest age is at 5-10 years. This could be causing that CAUTI is the most common nosocomial infection in the world reaching $30-40 \% .{ }^{6}$ Thus, it returns to whether medical personnel have performed the right catheter placement and treat in a sterile area. Because nosocomial occur most often when the surrounding environment is not sterile.

The results in this study found that there was an association between gender and bacteria colony count in CAUTI patients urine's culture, with $p<0.05$ ( $p=0.020)$. It can be concluded that gender and bacteria colony count in urine culture has significant relation. A study in India found that $p$-value of 0.471 , which means there was no significant relation. ${ }^{6}$ Data obtained in this research is described in 23 female subjects had urine culture $\geq 10^{5}$ from 54 subjects $(42.6 \%)$. In the literature stated that female had significant relation with positive urine culture $\left(\geq 10^{5}\right)$, this is caused by female anatomy which has shorter urethra than male and closer to the perineum, and vagina so that bacteria are more easily enter the body. ${ }^{7}$ So that the results of the Chi-square test in this study are not in accordance with the results of literature.

In the first year of life, urinary tract incidence in male is more common than female. ${ }^{8}$ In this research found that male incidence rate in first year was $14(60.8 \%)$, whereas female was $9(39.2 \%)$, then we can conclude that this research results is in accordance with the literature.

A study in America showed that gender is an important risk factor in CAUTI occurrence, female has higher risk more than female because of anatomy differences, especially in genital area such as shorter urethra. ${ }^{6}$ In this research was obtained equal sample, 28 male $(51.8 \%)$ and 26 females (48.2\%). This research is not in accordance with the literature, this incompatibility could be cause due to the small sample in this study. ${ }^{9,10}$
From this research results found that 54 subjects had same clinical symptoms which were fever $>38^{\circ} \mathrm{C}$, with various age range, urinary tract infection attacked all ages and specifically infant till 1 year. The clinical symptom can be fever. In higher age range till 4 years, high fever can be occurred to cause seizures, diarrhea, even dehydration. In children, the clinical symptoms are usually reduced and lighter, then start to show local urinary tract infection clinical symptoms such as polacisuria, dysuria, urgency, frequency and bedwetting, while stomachache, waist pain and pyrexia are less often found. ${ }^{5}$ This research results showed 54 subjects $(100 \%)$ had fever and 45 subjects among them had suprapubic tenderness (83.3\%). From 4452 children, $67 \%$ of them had fever symptoms and obtained $p<0.003$ which can be concluded that there was significant relation between fever and urinary tract infection. ${ }^{7}$ This research is in accordance with the literature.

The results of this research were found that patients who used catheter for 3-5 days had the highest CAUTI incidence and followed by 6-8 days. A literature found that the incidence in patient who used catheter for 6-9 days was $60 \%$ and patient who used catheter longer than 10 days had incidence of $93 \%$. Therefore, the risk of CAUTI is high at the duration of using catheter. ${ }^{9}$ The catheter usage duration is a significant factor to predict CAUTI occurrence. The most influential risk for urinary tract infections is due to catheter placement itself or catheter abuse. The duration of catheter use is the highest factor in the CAUTI diagnosis. As time goes by then the risk of CAUTI is increase around 3\%$10 \% .{ }^{11}$ On other hand, CAUTI incidence in first week were higher (37.6\%) and second week (27.7\%). ${ }^{12}$ If this research results following previous literature ${ }^{9}$, thus there will be no accordance, but if following another literature ${ }^{12}$ then these research results are compatible with the literature.

Results of this study found that the most causative bacteria of CAUTI is Escherichia coli at 24 subjects (44.4\%) continued with Klebsiella pneumoniae at 9 subjects (16.7\%). It was found that $22 \%$ Escherichia coli and $18 \%$ Klebsiella pneumoniae in experts' study. ${ }^{5}$ This research also conducted by NHSN that got the most causative bacteria in CAUTI was Escherichia coli as many as $21 \%$.

These research results were found that MDRO classified bacteria as many as 26 subjects (48\%) from 54 positive urine culture, it was obtained that the highest number of bacteria was in 16 subjects Escherichia coli ESBL positive (61.5\%), 5 subjects Klebsiella pneumoniae ESBL positive (19.2\%), 2 subjects Acinetobacter baumannii 
positive (7.7\%), 1 subject Klebsiella oxytoca ESBL positive $(3.8 \%)$, and 1 subject Pseudomonas aeruginosa positive $(3.8 \%)$, and 1 subject $M R S A$ positive (3.8\%). From 26 subjects, $79.3 \%$ among them were gram-negative bacteria. Most study also found that gram-negative bacteria are the most commonly found from all MDROs in hospitals. Therefore, can be concluded that most bacteria in this research and literature are the same, namely gram-negative bacteria.

This study faced some limitations and difficulties, however further study with more samples recruitment is necessary to gain more consistent the results.

\section{Conclusion}

From the positive urine culture results of children using urinary catheter. As much as $38.5 \%$ percent were due to CAUTI. Escherichia coli and Klebsiella pneumonia were the most common bacteria causing CAUTI and there is a significant association between gender and the number of bacterial colonies. Incident rate could not be calculated because the denumerator cannot be found, therefore the CAUTI incidence rate at Dr. Soetomo General Hospital in 2017 were 54.

\section{References}

1. World Health Organization [Internet]. The burden of health careassociated infection worldwide [cited 2018 Nov 21]. Available from: http://www.who.int/infection-prevention/publications/ burden_hcai/en/.

2. Chenoweth C, Saint S. Preventing catheter-associated urinary tract infections in the intensive care unit. Crit Care Clin. 2013; 29(1): 19-32.

3. Garner JS, Jarvis WR, Emori TG, Horan TC, Hughes JM. CDC definitions for nosocomial infections, 1988. Am J Infect Control. 1988; 16(3): 128-40.

4. Apisarnthanarak A, Thongphubeth K, Sirinvaravong S, Kitkangvan D, Yuekyen C, Warachan B, et al. Effectiveness of multifaceted hospitalwide quality improvement programs featuring an intervention to remove unnecessary urinary catheters at a tertiary care center in Thailand. Infect Control Hosp Epidemiol. 2007; 28(7): 791-8

5. Simon AK, Hollander GA, McMichael A. Evolution of the immune system in humans from infancy to old age. Proc Biol Sci. 2015; 282(1821): 20143085. doi: 10.1098/rspb.2014.3085.

6. Warren JW. Catheter-Associated urinary tract infection. Int J Antimicrob Agents. 2001; 17(4): 299-303.

7. Leelakrishna P, Karthik Rao B. A study of risk factors for catheter associated urinary tract infection. Int J Adv Med. 2018; 5(2): 334-9.

8. Johnson CE. New advances in childhood urinary tract infections. Pediatr Rev. 1999; 20(10): 335-42.

9. Vinoth M, Prabagaravarthanan R, Bhaskar M. Prevalence of microorganisms causing catheter associated urinary tract infections (CAUTI) among catheterised patients admitted in a tertiary care hospital. Int J Res Med Sci. 2017; 5(6): 2367-72.

10. Shaw KN, Gorelick M, McGowan KL, Yakscoe NM, Schwartz JS Prevalence of urinary tract infection in febrile young children in the emergency department. Pediatrics. 1998; 102(2): e16. doi: 10.1542/ peds.102.2.e16.

11. Saint S, Lipsky BA, Goold SD. Indwelling urinary catheters: a onepoint restraint? Ann Intern Med. 2002; 137(2):125-7.

12. Jayasukhbhai DM, Komal DP, Vegad MM. Study of incidence and risk factors of urinary tract infection in catheterised patients admitted at tertiary care hospital. Int J Res Med Sci. 2015; 3(12): 3808-11. 